\title{
ARTICLE
}

Received 8 Sep 2014 | Accepted 1 Apr 2015 | Published 12 May $2015 \quad$ DOl: 10.1038/ncomms8078

\section{Hepatic insulin signalling is dispensable for suppression of glucose output by insulin in vivo}

\author{
Paul M. Titchenell', Qingwei Chu', Bobby R. Monks ${ }^{1} \&$ Morris J. Birnbaum ${ }^{1, \dagger}$
}

Insulin signalling and nutrient levels coordinate the metabolic response to feeding in the liver. Insulin signals in hepatocytes to activate Akt, which inhibits Foxo1 suppressing hepatic glucose production (HGP) and allowing the transition to the postprandial state. Here we provide genetic evidence that insulin regulates HGP by both direct and indirect hepatic mechanisms. Liver-specific ablation of the IR (L-Insulin Receptor KO) induces glucose intolerance, insulin resistance and prevents the appropriate transcriptional response to feeding. Liver-specific deletion of Foxo1 (L-IRFoxo1DKO) rescues glucose tolerance and allows for normal suppression of HGP and gluconeogenic gene expression in response to insulin, despite lack of autonomous liver insulin signalling. These data indicate that in the absence of Foxo1, insulin signals via an intermediary extrahepatic tissue to regulate liver glucose production. Importantly, a hepatic mechanism distinct from the IR-Akt-Foxo1 axis exists to regulate glucose production.

\footnotetext{
${ }^{1}$ Department of Medicine, Institute for Diabetes, Obesity, and Metabolism, Perelman School of Medicine at the University of Pennsylvania, Philadelphia, Pennsylvania, USA. $\dagger$ Present address: CVMED, Pfizer Inc., Cambridge, MA. Correspondence and requests for materials should be addressed to M.J.B. (email: birnbaum@mail.med.upenn.edu).
} 
T he dynamic regulation of liver glucose metabolism is essential for systemic carbohydrate homeostasis and organismal survival. During times of starvation, the liver produces the amount of glucose necessary to meet the metabolic demands of the body. This increased hepatic glucose production (HGP) during fasting results from an initial breakdown of glycogen stores before transitioning to gluconeogenesis from various precursors. In the postprandial state, this process is opposed by the rise of the hormone insulin, which suppresses HGP. However, in insulin-resistant disorders such as diabetes, insulin fails to regulate HGP, leading to elevated circulating glucose concentrations ${ }^{1,2}$. Various models have been proposed to explain the inability of insulin to suppress HGP during diabetes, though there is still no consensus for the mechanism. Prevalent hypotheses include increased delivery of gluconeogenic precursors and fatty acids to the liver, accumulation of neutral lipids in liver, altered systemic cytoadipokines, distorted glucagon-to-insulin ratios, and defective hepatic insulin signalling.

Since excess HGP drives fasting hyperglycaemia in diabetes, the elucidation of the mechanisms of how insulin regulates hepatic metabolism in normal and pathological livers has received substantial attention. In liver, insulin signals through its receptor (IR), insulin receptor substrates (Irs) and phosphoinositide $3^{\prime}$-kinase to activate the serine/threonine kinase Akt, which utilizes several distinct downstream pathways to modulate liver metabolism ${ }^{4}$. In hepatocytes, insulin promotes protein translation and cell growth by activating the mammalian target of rapamycin complex 1 (mTORC1) via Akt-mediated phosphorylation and inactivation of the tuberous sclerosis protein 1 and 2 complex ${ }^{5}$. Insulin also induces lipogenesis and glycogen synthesis via Akt-dependent mechanisms ${ }^{6-8}$. Finally, insulin stimulates an Akt-dependent inhibitory phosphorylation of Foxol, which is thought to be the master regulator of key gluconeogenic genes leading to the subsequent regulation of glucose output ${ }^{9}, 10-14$.
In support of this notion, ablating hepatic Foxo1 in insulinresistant models improves glucose homeostasis ${ }^{15,16}$. However, deletion of liver Foxo1 in lean mice only modestly reduces fasting glucose and hypoglycemia occurs only after prolonged fasting, leading to uncertainty concerning the role of Foxo1 in normal liver glucose metabolism ${ }^{11,17}$.

A reduction of the IR-IRS-Akt pathway activity in mouse livers results in glucose intolerance, systemic insulin resistance and a failure to suppress appropriately glucose production in response to insulin 15,17,18. Concomitant deletion of Foxo1 normalizes the glucose intolerance and hyperinsulinemia triggered by hepatic deletion of Irs or $A k t^{15,17}$. Surprisingly, mice with liver-specific deletion of Akt and Foxo1 (L-AktFoxolTKO) still respond to insulin in vivo by suppressing gluconeogenic gene expression and HGP. Furthermore, L-AktFoxolTKO mice adapt appropriately to the postprandial state despite lacking canonical liver insulin signalling ${ }^{17}$. These data are inconsistent with the established model of hepatic insulin action and suggest Akt is not an obligate intermediate for insulin action under all conditions. Importantly, these data suggest the existence in liver of a signalling pathway parallel to the Akt-Foxo1 axis that is capable of regulating HGP. At least two alternative mechanisms can be formulated to explain the insulin effects in L-AktFoxo1TKO mice: (1) insulin acts directly on the liver via the IR-Irs pathway; however, a bifurcation occurs before Akt thereby activating an unidentified parallel pathway independent of Akt to suppress $\mathrm{HGP}^{19}$; or (2) insulin acts non-autonomously via a peripheral tissue to regulate HGP.

In the study reported in this manuscript, we tested the second hypothesis by deleting insulin receptor specifically and exclusively in liver, with and without concomitant deletion of Foxo1. We reasoned that if insulin were suppressing HGP by acting via an intermediary, non-hepatic cell type, which then sends a signal to liver, the effect of insulin should not depend on the presence of its receptor on hepatocytes. a

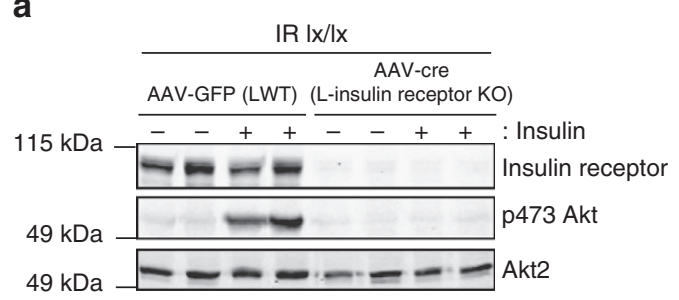

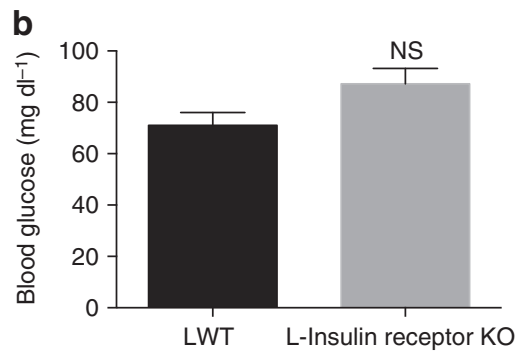

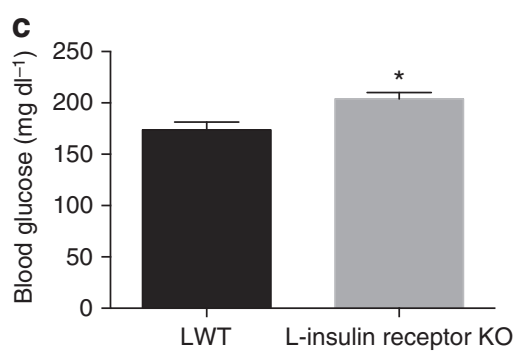

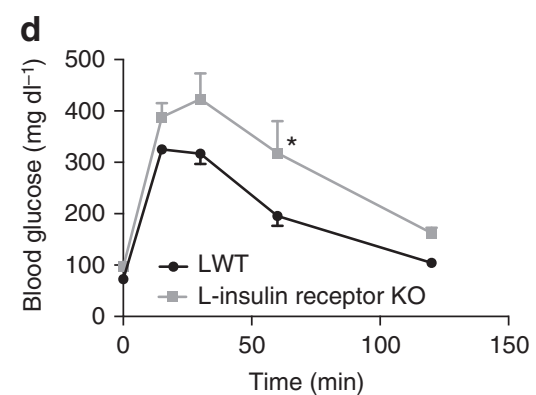

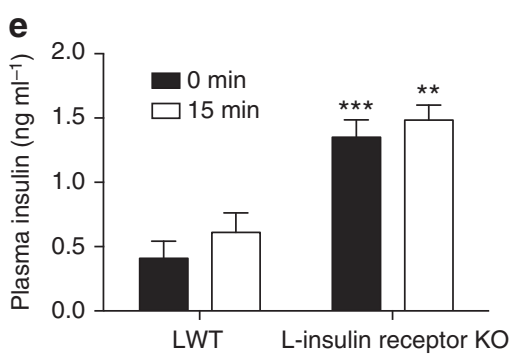

Figure 1 | Acute deletion of liver insulin receptor leads to glucose intolerance and insulin resistance. (a) Western blot of primary hepatocytes from mice treated with $10 \mathrm{nM}$ insulin for $15 \mathrm{~min}$ and probed for specific proteins as indicated. AAV-GFP $=\mathrm{LWT}$ mice, AAV-Cre $=\mathrm{L}-\mathrm{Insulin}$ Receptor KO mice. $(\mathbf{b}, \mathbf{c})$ Blood glucose concentrations in mice after overnight fast $(\mathbf{b})$ and overnight fasted following by $4 \mathrm{~h}$ refeeding of normal chow (c). NS, not significant ${ }^{\star} P<0.05$. $n=5$ mice. (d) Intraperitoneal glucose tolerance test $\left(2 \mathrm{~g} \mathrm{~kg}^{-1}\right)$ on overnight fasted mice. $n=5$ mice per group. ${ }^{\star} P<0.05$ versus LWT. Statistical analysis was performed two-way analysis of variance followed by Bonferroni when two conditions were involved. (e) Insulin levels before and after 15 min following glucose injection IP. $n=5$ mice per group. ${ }^{\star \star \star} P<0.001,{ }^{\star \star} P<0.01$ versus LWT. All the data are presented as mean \pm s.e.m. Statistical analysis was performed using Students's $t$-test followed by two-tailed analysis. A $P<0.05$ was considered statistically significant. 


\section{Results}

Acute liver-specific deletion of IR. To minimize potential secondary and/or compensatory responses from congenital hepatic insulin receptor (IR) deficiency, we generated a model of acute, liver-specific knockout of the $I R$. Adult $I R^{\operatorname{lox} P / l o x P}$ mice were injected with an adeno-associated virus containing a liverspecific promoter (thyroxine-binding globulin) driving Cre recombinase to delete the $I R$ (L-Insulin Receptor $\mathrm{KO})^{8} . I R^{\text {loxP } / \text { lox } P}$ (LWT) mice injected with virus expressing GFP instead of Cre served as a control. A western blot confirmed efficient deletion of $I R$ in primary hepatocytes isolated from mice following the virus injection (Fig. 1a). Akt phosphorylation at Ser473 in response to insulin was undetectable in hepatocytes isolated from L-Insulin Receptor $\mathrm{KO}$ mice. In contrast to the major metabolic abnormalities reported in the congenital $I R$ liver knockout mouse (LIRKO) ${ }^{18}$, L-Insulin Receptor KO mice displayed normal fasting glucose levels (Fig. 1b) and modest postprandial hyperglycaemia following $4 \mathrm{~h}$ of refeeding (Fig. 1c). As expected, L-Insulin Receptor KO mice were glucose intolerant (Fig. 1d) and hyperinsulinemic following fasting and $15 \mathrm{~min}$ following an intraperitoneal glucose injection (Fig. 1e).

Hepatic Foxo1 deletion in L-Insulin Receptor KO mice. Insulin signals via its receptor to activate Akt and suppress Foxo1dependent transcription. Hepatic Foxol deletion reverses the metabolic abnormalities resulting from liver-specific knockout of Irs or $A k t^{15,17}$. To determine whether constitutive Foxol activation is responsible for the abnormal glucose metabolism following hepatic IR deletion, adult $I R^{\text {loxP/loxP }} ;$ FoxO $1^{\text {loxP/loxP }}$ mice were injected with AAV-Tbg-Cre to generate liver-specific deletion of IR and Foxo1 (L-IRFoxo1DKO). Mice injected with virus expressing GFP instead of Cre served as controls (LWT). A western blot confirmed excision of the $I R$ and Foxo 1 in primary hepatocytes and liver lysates from L-IRFoxo1DKO mice (Fig. 2a). L-Insulin Receptor $\mathrm{KO}$ and L-IRFoxo1DKO mice failed to activate canonical insulin targets (phosphorylation of Akt, S6 and PRAS40) following a supraphysiological dose of insulin (Fig. 2b). However, feeding elicited significant S6 phosphorylation in all genotypes, supporting the notion that hepatic IR is not required for the activation of the mTorcl complex by nutrients (Fig. 2c) ${ }^{20}$. Concomitant deletion of Foxo1 restored Igfbp1 protein to LWT levels, while also partially increasing glucokinase protein concentration albeit not to the levels present in LWT mice (Fig. 2c). Deletion of Foxo1 normalized the fasting (Fig. 2d) and postprandial hyperglycaemia in the L-Insulin Receptor KO (Fig. 2e). In addition, L-IRFoxo1DKO mice demonstrated improved glucose tolerance (Fig. 2f) and Foxo1 deletion rescued the hyperinsulinemia caused by hepatic deletion of the $I R$ (Fig. 2g). The relatively normal metabolic phenotype of the L-IRFoxo1DKO mice was assessed in an alternative model, that is, the congenital liver-specific knockout generated with a liver-specific Cre transgene. Consistent with data with acute recombination, deletion of Foxo1 normalized the glucose intolerance caused by liver-specific deletion of $I R$ (Fig. 2h). These data support the concept that a significant increase in Foxol activity in the L-Insulin Receptor $\mathrm{KO}$ mice as a result of defective Akt activation is responsible for the diabeticlike phenotype of the L-Insulin Receptor KO mice.

Insulin suppresses HGP in vivo in L-IRFoxo1DKO mice. Despite the lack of hepatic insulin signalling in L-IRFoxo1DKO, the wild-type fasting glucose and insulin levels suggest these mice have normal HGP. However, these data do not provide information about the ability of insulin to regulate HGP. To ascertain whole-body insulin sensitivity directly and determine if insulin can suppress HGP independent of liver insulin signalling, hyperinsulinemic-euglycemic clamps in awake, unrestrained mice were performed. During insulin infusion under glucose clamp conditions, L-Insulin Receptor KO mice displayed a lower glucose infusion rate (Fig. 3a), a 25\% decrease in glucose disposal (Fig. 3b) and a failure to suppress hepatic glucose production (Fig. $3 \mathrm{c}, \mathrm{d}$ ) compared with LWT mice. Concomitant deletion of Foxo1 in L-IRFoxo1DKO mice normalized the glucose infusion rate (Fig. 3a) confirming that Foxo1 deletion reversed the insulin resistance conferred by loss of the IR. L-IRFoxo1DKO mice had a normal rate of glucose disposal (Fig. 3b) and, most importantly, insulin suppressed hepatic glucose production to the same extent as in LWT mice (Fig. 3c,d). As noted previously, in this hyperinsulinemic-euglycemic clamp paradigm, insulin reduced glucose-6-phosphatase (G6pc) without affecting phosphoenolpyruvate carboxykinase (Pck1) expression in wild-type mice (Fig. 3f) ${ }^{6}$. At the end of the clamp procedure, levels of G6pc and $P c k 1$ in L-Insulin Receptor KO mice were significantly elevated compared with both LWT and L-IRFoxo1DKO mice (Fig. 3g). The data support the hypothesis that insulin signals via an extrahepatic tissue to regulate hepatic glucose production and the expression of G6pc.

Feeding suppresses gluconeogenic genes in L-IRFoxo1DKO mice. L-IRFoxo1DKO mice are glucose tolerant and insulin suppresses hepatic glucose production despite lacking hepatic insulin signalling. These data support previous observations that mice lacking Akt isoforms and Foxol in the liver can respond to insulin to reduce glucose production and transition to the fed state $^{17}$. In addition to rescuing the defects in glucose metabolism, deletion of Foxo1 restored the inability of both the Irs and Akt liver double knockouts to regulate genes involved in gluconeogenesis ${ }^{15,17}$. To test if hepatic insulin action is required for the changes in gene expression in response to feeding, mice were subjected to an overnight fast and refed. In LWT mice, $4 \mathrm{~h}$ after refeeding there was the expected gene changes in expression, with suppression of $G 6 p c, P c k 1, I g f b p 1$ and induction of Srebplc and Gck (Fig. 4). Similar to previous models of defective hepatic insulin action, L-Insulin Receptor KO mice failed to reduce G6pc, $P c k 1$ or increase Srebp1 and Gck mRNA in response to feeding; in addition, there was augmented expression of the Foxol target genes Igfbp1 and Irs2 (Fig. 4). Despite the loss of hepatic insulin signalling in L-IRFoxo1DKO mice, refeeding suppressed both G6pc and Pck1 expression but failed to induce Srebp1 and Gck expression (Fig. 4). Thus, hepatic insulin signalling is dispensable for the normal prandial response on gluconeogenic genes yet is required for the induction of Gck and the master lipogenic transcription factor, Srebp1c.

Insulin fails to suppress genes in vitro in L-IRFoxo1DKO mice. To confirm that insulin was acting cell-non-autonomously to regulate HGP and gluconeogenic genes in L-IRFoxo1DKO mice, primary hepatocytes were isolated from LWT, L-Insulin Receptor $\mathrm{KO}$ and L-IRFoxo1DKO mice. Glucose production was measured over $6 \mathrm{~h}$ in response to db-cAMP, insulin and db-cAMP and insulin. In all genotypes, db-cAMP stimulated glucose production approximately 30-40\% (Fig. 5a). L-Insulin Receptor KO mice exhibited increased glucose production that was normalized with concomitant Foxo1 deletion during all treatments (Fig. 5a). G6pc and Pck1 expression was induced in response to db-cAMP in both LWT and L-Insulin Receptor KO. However, L-IRFoxo1DKO mice exhibited a defect in gluconeogenic gene expression in response to db-cAMP (Fig. 5b). Insulin did not affect glucose production in isolated hepatocytes in any genotype or condition. However, insulin significantly reduced $G 6 p c, P c k 1$ and $I g f b p 1$ 
a

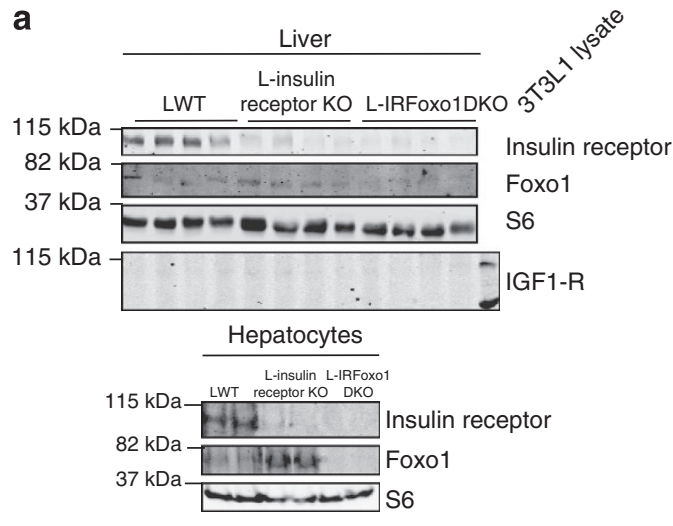

C

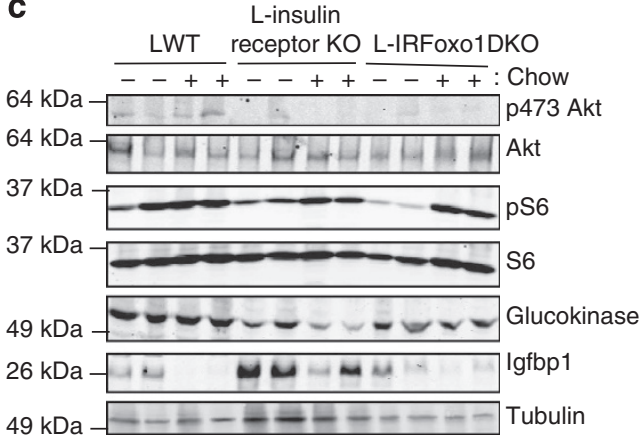

b

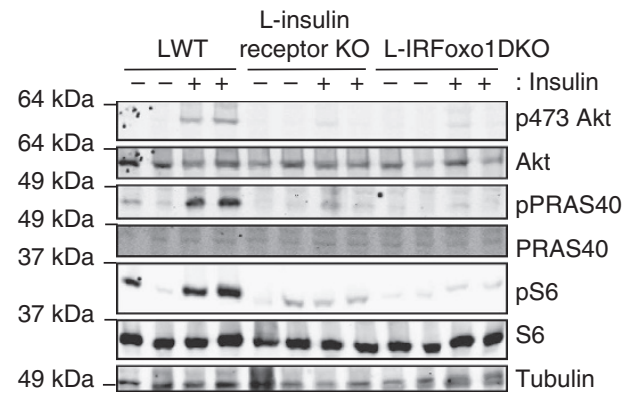

d
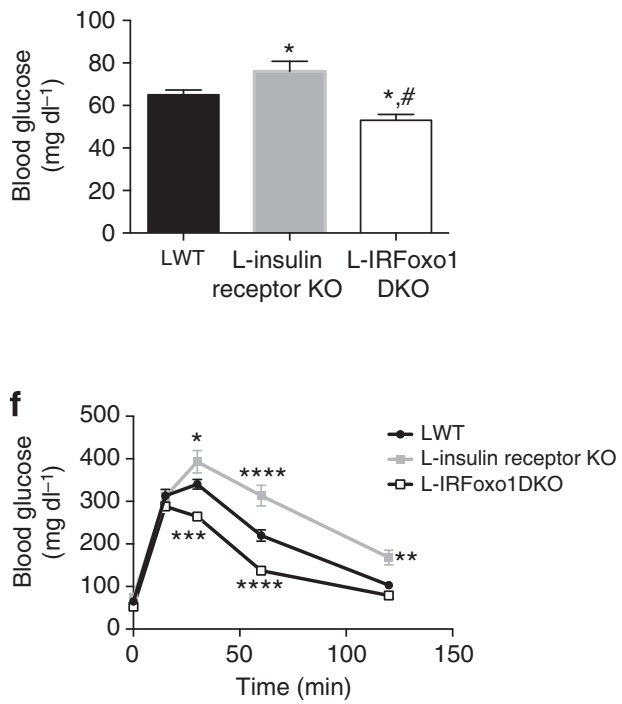

h

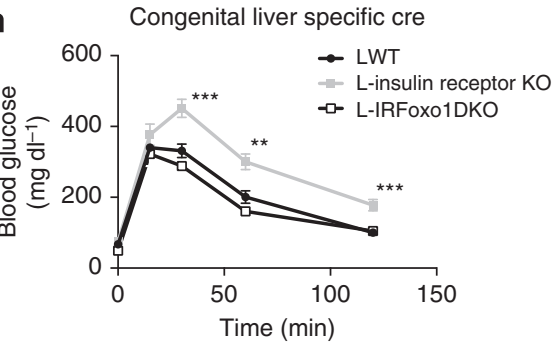

Figure 2 | Liver Foxo1 deletion normalizes glucose levels and hyperinsulinemia in L-Insulin Receptor KO mice despite lack of hepatic insulin signalling. (a) Western blot from primary hepatocyte and liver lysates of mice from indicated gentoypes probed for specific proteins as indicated. (b) Western blot of liver lysates in mice fasted overnight and i.p. injected with either saline or insulin $\left(2 \mathrm{mUg}^{-1}\right)$ and probed for specific proteins as indicated. (c) Western blot of liver lysates in mice fasted overnight and refed normal chow for $4 \mathrm{~h}$ and probed for specific proteins as indicated. (d,e) Blood glucose concentrations in mice after overnight fast (d) and overnight fasted following by $4 \mathrm{~h}$ refeeding of normal chow $(\mathbf{e}) .{ }^{\star} P<0.05$ versus LWT, $\# P<0.0001$ versus L-Insulin Receptor KO. LWT group includes total of 18 mice in fasting group with $n=10 I R^{\text {loxP/loxP }}$ and $n=8 I R^{\text {loxP/loxP }} ;$ FoxolloxP/loxP and in refed group includes total of 9 mice in fasting group with $n=4 \mathbb{I}^{\text {loxP/loxP }}$ and $n=5 I^{\text {loxP/loxP }} ;$ Foxo $1^{\text {loxP/loxP }}$. L-Insulin Receptor KO includes $n=12$ for fasting group and $n=6$ for refed group. L-IRFoxo1DKO includes $n=22$ for fasting group and $n=7$ for refed group. (f) Intraperitoneal glucose tolerance test $\left(2 \mathrm{~g} \mathrm{~kg}^{-1}\right)$ on overnight fasted

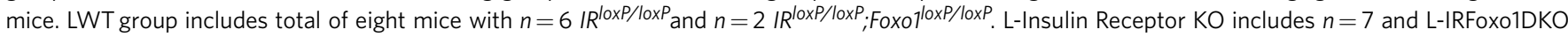
includes $n=8$. ${ }^{\star} P<0.05$ versus LWT, ${ }^{\star \star \star \star \star} P<0.0001$ versus LWT $(\mathbf{g})$ Insulin levels before and after 15 min following glucose injection. LWT group includes

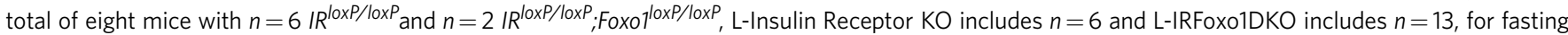
group. LWT group includes total of three mice with $n=2 I R^{\text {loxP/loxP }}$ and $n=1 / R^{\text {loxP/loxP }} ;$ FoxolloxP/loxP, L-Insulin Receptor KO includes $n=3$ and L-IRFoxo1DKO includes $n=8$ for 15 min group. ${ }^{\star \star \star \star} P<0.0001$ versus LWT, \# to $P<0.0001$ vs L-Insulin Receptor KO (h) Intraperitoneal glucose tolerance test ( $2 \mathrm{~g} k \mathrm{~kg}^{-1}$ ) on overnight fasted mice. $n=7-10$ mice. LWT group includes total of six mice with $n=5 / R^{\text {loxP/loxP }}$ and $n=1 / R^{\text {loxP/loxP }} ;$ Foxo ${ }^{\text {loxP/loxP }}$. L-Insulin Receptor KO includes $n=8$ and L-IRFoxo1DKO includes $n=5$. ${ }^{\star \star} P<0.01$ versus LWT, ${ }^{\star \star \star \star} P<0.0001$ versus LWT. All data are presented as mean \pm s.e.m. Statistical analysis was performed using one-way analysis of variance followed by Tukey post-test when more than two groups were compared, two-way analysis of variance followed by Bonferroni when two conditions were involved. A $P<0.05$ was considered statistically significant. 

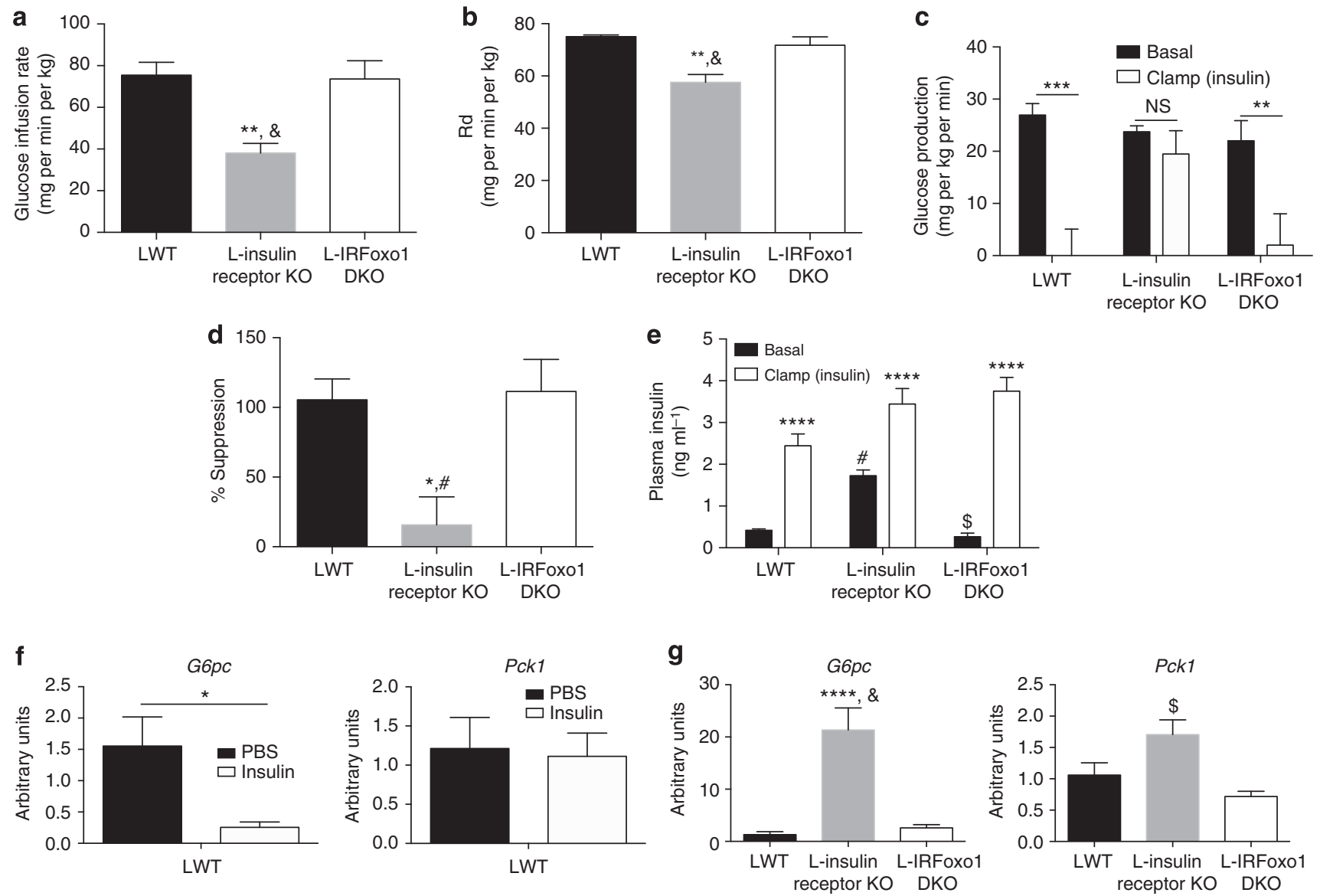

Figure 3 | L-IRFoxo1DKO are insulin responsive during euglycemic clamps. Hyperinsulinemic-euglycemic clamps were perfromed on unrestrained $5 \mathrm{~h}$ fasted mice using a $2.5 \mathrm{mU}$ ming ${ }^{-1} \mathrm{~kg}^{-1}$ infusion of insulin. (a) Steady state glucose infusion rate (b) rate of glucose disposal (c) hepatic glucose production during basal and insulin portions of the clamp (d) percent suppression of hepatic glucose production during the clamp portion compared to basal period (e) insulin levels during during basal and insulin portions of the clamp. ${ }^{\star} P<0.05$ versus LWT, ${ }^{\star \star} P<0.01$ versus $L W T$, ${ }^{\star \star \star} P<0.001$ versus $L W T$, ${ }^{\star \star \star \star} P<0.0001$ versus LWT \#P<0.05 versus Insulin Receptor KO, $\& P<0.01$ versus L-Insulin Receptor KO, $\$ P<0.0001$ versus L-Insulin Receptor KO.

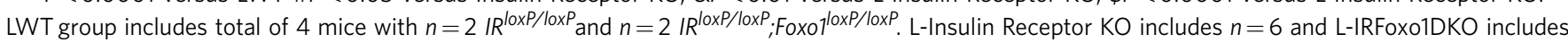
$n=4$. ${ }^{\star \star \star \star} P<0.0001$ versus LWT $\$ P<0.0001$ versus L-Insulin Receptor KO. (f) Relative expression of G6pc and Pck1 under euglycemic conditions with/without infusion of $2.5 \mathrm{mU}$ ming ${ }^{-1} \mathrm{~kg}^{-1}$ of insulin for $120 \mathrm{~min} .{ }^{\star} P<0.05$ versus PBS of indicated genotype. $n=3$ (PBS), $n=6$ (insulin). (g) Relative expression of G6pc and Pck1 under euglycemic conditions with infusion of $2.5 \mathrm{mU}$ ming ${ }^{-1} \mathrm{~kg}^{-1}$ of insulin for 120 min. LWT group includes total of 4 mice

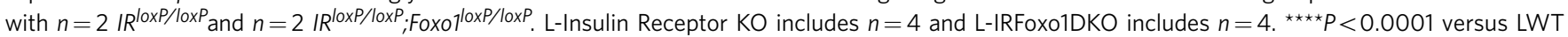
$\$ P<0.0001$ versus L-Insulin Receptor KO. All data are presented as mean \pm s.e.m. Statistical analysis was performed using one-way analysis of variance followed by Tukey post-test. A $P<0.05$ was considered statistically significant.

mRNA in hepatocytes isolated from LWT but not from L-Insulin Receptor KO or L-IRFoxo1DKO mice.

\section{Discussion}

In this study, we used genetic loss of function experiments in mice to test the requirement for hepatic insulin action for the insulin-dependent regulation of glucose metabolism and transcription in mouse liver. Data presented herein confirm previous studies that signalling via the hepatic insulin receptor is essential for normal glucose metabolism in adult mice ${ }^{18}$. Substantial data support a model in which insulin regulates glucose metabolism by signalling through Akt-mediated inactivation of the transcription factor, Foxo1, thus contributing to the metabolic transition that accompanies the intake of food. Nonetheless, insulin suppressed hepatic glucose output during euglycemic clamps in mice with livers deficient for the IR and Foxol. These data refute the idea that the canonical model of hepatic insulin action in liver represents the exclusive pathway by which the hormone suppresses hepatic glucose output. Moreover, the persistence of hepatic effects of insulin in L-IRFoxo1DKO mice strongly supports the hypothesis that, at least under some conditions, insulin can act cell non-autonomously to suppress hepatic glucose output and regulate gene expression ${ }^{17}$. The hypothesis that indirect mechanisms contribute to the regulation of insulin mediated suppression of HGP dates back almost 60 years ${ }^{21}$. In support of this idea, restoring hepatic insulin signalling is not sufficient to fully suppress HGP in mice with systemic loss of the insulin receptor ${ }^{22}$. Data presented herein provide genetic evidence that insulin uses both an intra- and extrahepatic mechanisms to regulate liver metabolism.

Previous studies using liver-specific insulin receptor knockout (LIRKO) mice have reported robust defects in glucose homeostasis and peripheral insulin resistance ${ }^{18,23}$. LIRKO mice develop profound insulin resistance as adults; however, the lack of the insulin receptor from birth also leads to liver failure in aged mice. To avoid the complications of congenital liver-specific deletion, we employed a model that relies on acute deletion of the insulin receptor in adult mice ${ }^{8}$. L-Insulin Receptor $\mathrm{KO}$ mice displayed mild glucose intolerance and insulin resistance without 

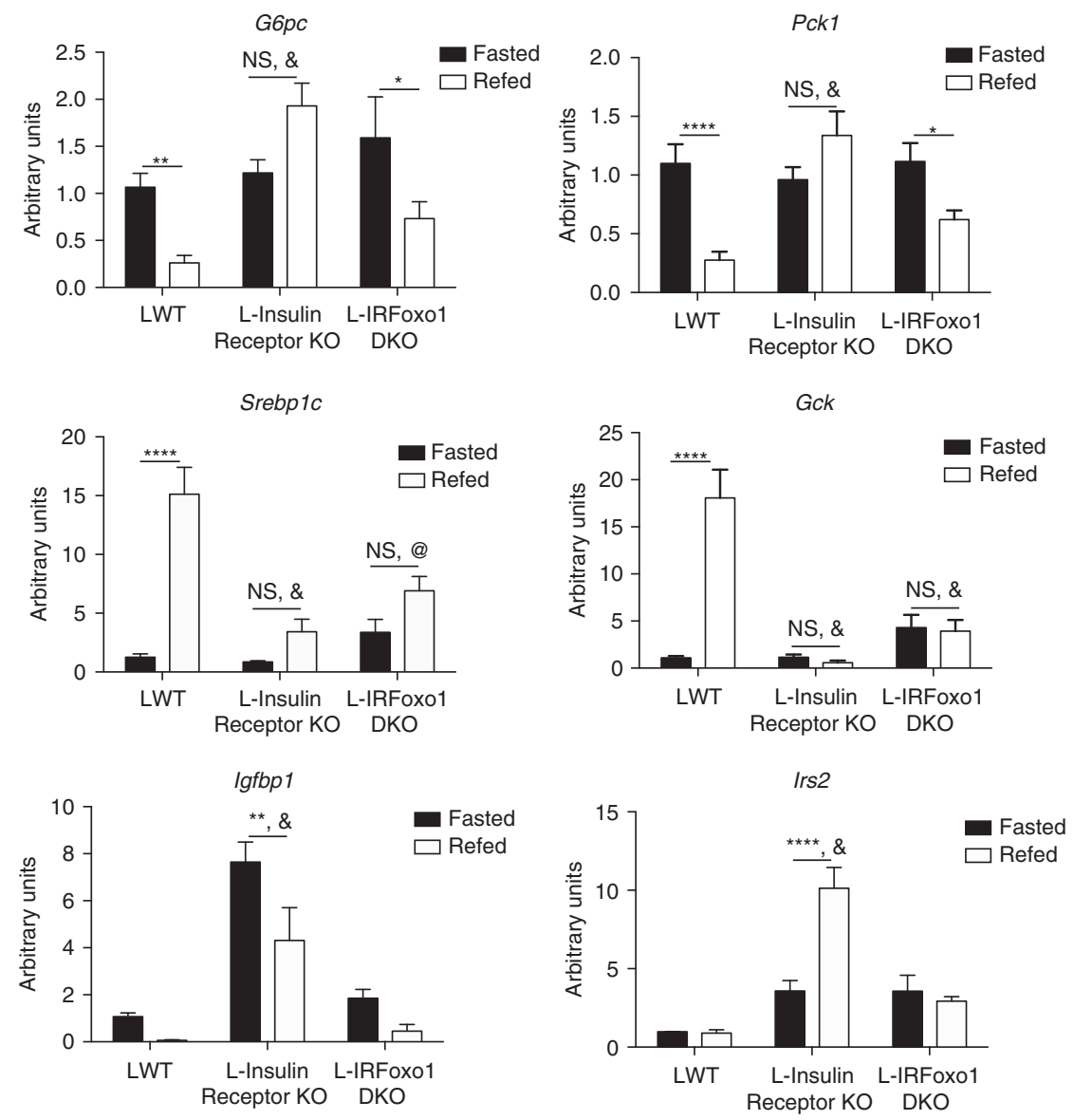

Figure 4 | Metabolic transcriptional response to feeding in the absense of hepatic insulin signaling. Hepatic gene expression in LWT, L-Insulin Receptor $\mathrm{KO}$, and L-IRFoxo1DKO following an overnight fast and $4 \mathrm{~h}$ of refeeding normal chow. LWT group includes total of 9 mice with $n=5 I R^{\text {loxP/loxP }}$ and $n=4$

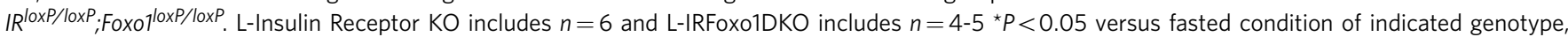
${ }^{\star \star} P<0.01$ versus fasted condition of indicated genotype, ${ }^{\star \star \star \star} P<0.0001$ versus fasted condition of indicated genotype, $\# P<0.0001$ versus fasted LWT, @ $P<0.001$ versus fed LWT. All data are presented as mean \pm s.e.m. Statistical analysis was performed using two-way analysis of variance followed by Bonferroni post-test. A $P<0.05$ was considered statistically significant.

the confounding peripheral insulin resistance and pronounced fasting hyperglycaemia in the LIRKO mice. Moreover, the mild hyperglycaemia of the acute IR deletion was less severe than in acute liver-specific deletion of both Akt isoforms ${ }^{17}$. This difference in severity may be due to significant basal Akt activity independent of insulin action, leading to partial inhibition of Foxol, even in fasting, non-obese mice ${ }^{17}$.

Considerable data refute the idea that insulin signals though a hepatocyte, cell autonomous pathway in L-IRFoxo1DKO mice. Insulin failed to promote phosphorylation of the canonical targets Akt and S6 in L-Insulin Receptor KO and L-IRFoxo1DKO mice. Though it has been suggested that IGF1-R can be induced in liver by stresses such as regeneration ${ }^{24}$, we did not detect IGF1-R protein in livers from LWT, L-Insulin Receptor KO or L-IRFoxo1DKO mice (Fig. 2a). High concentrations of insulin were completely without effect on isolated hepatocytes lacking insulin receptors with or without concomitant deletion of Foxo1 (Fig. 5b).

One of the major unanswered questions that arise from this study is the identity of the extrahepatic tissue that is binding insulin and transmitting the signal to suppress hepatic glucose output and gluconeogenic gene expression. On the basis of recent work, perhaps the most likely site of insulin action is the central nervous system (CNS), which has been proposed to serve as an organizing centre for metabolic control ${ }^{25-27}$. In support of a direct communication between the CNS and liver, intracerebral ventricular infusion of insulin suppresses HGP without activation of hepatic insulin signalling through the hepatic vagus nerve ${ }^{28,29}$. Moreover, mice with insulin receptor deficiency in AgRP neurons fail to suppress HGP in response to peripheral insulin infusion during euglycemic clamps ${ }^{30}$. However, infusion of insulin in the brain of dogs only increases liver glycogen synthesis without affecting glucose output, questioning the significance of a CNS mechanisms in the regulation of $\mathrm{HGP}^{31,32}$. Future studies are needed to assess the contribution of insulin action in the CNS to the regulation of HGP in mice with livers lacking autonomous insulin action.

In addition to the CNS, a more classical target of insulin action is adipose tissue. It has been hypothesized that the insulindependent suppression of lipolysis limits gluconeogenesis by reduction in circulating glycerol and free fatty acids ${ }^{33}$. Preventing the fall of free fatty acids during hyperinsulinemic-euglycemic clamps by infusing a lipid/heparin mixture antagonizes the reduction of hepatic glucose output in $\operatorname{dogs}^{34}$. Moreover, pharmacological suppression of lipolysis alone is sufficient to reduce HGP independent of changes in insulin levels ${ }^{35}$. The other well-established pathway for regulating HGP is through the counter-regulatory hormone glucagon, whose secretion by the alpha cells of the pancreas is itself reduced by insulin ${ }^{3,36}$. Indeed, the decline in portal glucagon concentrations during hyperinsulinemic-euglycemic clamps in dogs correlates well with reductions in $\mathrm{HGP}^{37}$. However, a reduction in peripheral 
a

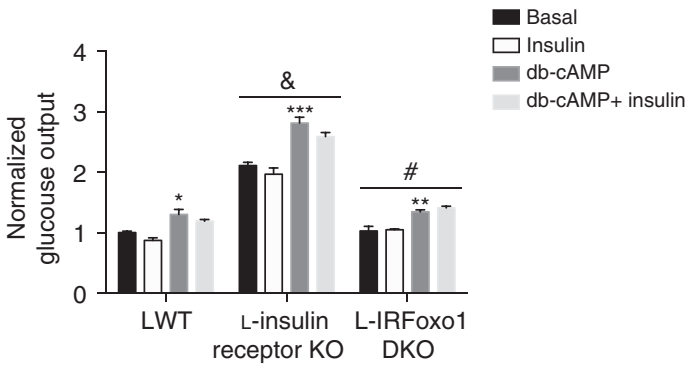

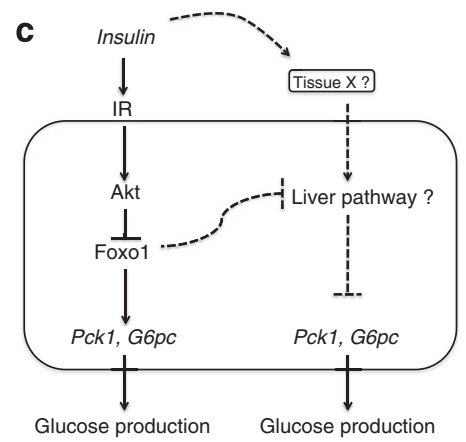
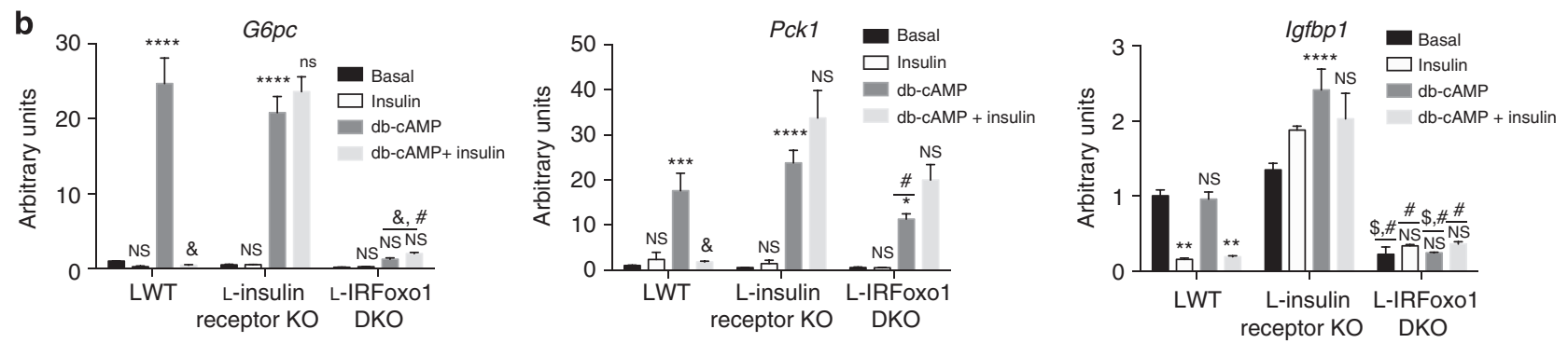

Figure 5 | Glucose production and gluconeogenic gene response to insulin and cAMP in isolated primary hepatocytes. Primary hepatocytes were isolated from indicated genotype and treated with either $0.1 \mathrm{mM}$ db-cAMP and/or $0.1 \mu \mathrm{M}$ insulin for $6 \mathrm{~h}$. (a) Glucose concentration in media was determined and expressed normalized to protein concentration. (b) Gene expression analysis was performed at the end of the treatments. Data is from a representative experiment and presented as mean \pm s.e.m.. ${ }^{\star} P<0.05$ versus basal condition of indicated genotype, ${ }^{\star \star} P<0.01$ versus basal condition of indicated genotype, ${ }^{\star \star \star} P<0.001$ versus basal condition of indicated genotype, ${ }^{\star \star \star \star} P<0.001$ versus basal condition of indicated genotype , $\# P<0.0001$ versus L-Insulin Receptor KO, \&P<0.0001 versus LWT for indicated treatment condition. All data are presented as mean \pm s.e.m. Statistical analysis was performed using two-way analysis of variance followed by Bonferroni post-test. A $P<0.05$ was considered statistically significant. (c) In hepatocytes, insulin stimulates the kinase Akt thus inactivating the transcription factor Foxo1, which leads to the suppression of gluconeogenic genes and glucose production. In addition to the canonical IR-Akt liver pathway, there is an insulin responsive non-hepatic tissue (tissue X) capable of communicating with the liver to regulate hepatic glucose output and gluconeogenic genes. An unidentified liver pathway receives the insulin signal via tissue $X$ to suppress gluconeogenic genes and glucose output in response to insulin. Deletion in liver of the IR, Irs, or Akt gene or high fat diet feeding leads to abberant Foxo1 activity, which represses this unidentified liver pathway. Ablation of hepatic Foxo1 alleviates this suppressive effect allowing insulin to regulate hepatic glucose metabolism.

glucagon during clamp experiments in mice has been difficult to observe $^{23}$. Defining the relative contributions of these cell nonautonomous pathways for the regulation of HGP by insulin will be of significant interest.

Since these data establish an extrahepatic pathway in the regulation of HGP, there must exist a liver autonomous mechanism to receive the signal initiated by insulin's interaction with a non-hepatic tissue. In L-Insulin Receptor KO mice, this unidentified liver autonomous pathway is suppressed by aberrant Foxo1 activity, preventing insulin's regulation of hepatic glucose output. On deletion of Foxol, as in L-IRFoxo1DKO mice, the suppressive effect of Foxol on this pathway is lost allowing insulin to signal to the liver via an intermediary peripheral tissue to suppress hepatic glucose output and gluconeogenic gene expression (Fig. 5c). The identity of this pathway remains unknown and presents a novel therapeutic target for the regulation of HGP independent of hepatic insulin action.

Though deletion of Foxo1 in IR null livers restored the response of gluconeogenic genes to nutritional regulation, lipogenic genes such as Srebplc and Gck remained unresponsive to refeeding in the absence of hepatic insulin receptors (Fig. 4). Following a meal, insulin and nutrients activate the mTORC1 signalling pathway that leads to increased lipogenesis and lipogenic gene expression, in part by inducing the transcription and posttranslational processing of SREBP $1 c^{8,38}$. However, refeeding fails to increase Srebplc and Gck gene expression in the liver of L-IRFoxo1DKO to the same extent as LWT mice. Indeed, the hepatic insulin receptor is required for SREBP1c induction and de novo lipogenesis in vivo ${ }^{20}$. The failure to induce lipogenic genes in L-IRFoxo1DKO mice suggests strongly that insulin can stimulate lipid synthesis only by a cell autonomous pathway.

In summary, we have demonstrated that the abnormal hepatic glucose metabolism and insulin resistance resulting from lack of hepatic insulin receptor is dependent on a constitutively active Foxo1. On Foxo1 deletion, the insulin-dependent regulation of glucose metabolism in vivo was largely restored, supporting the idea that reducing Foxol activity in insulin resistant disorders may have beneficial effects ${ }^{16,39}$. In addition, we provided genetic evidence that an extra-hepatic insulin-responsive tissue communicates with the liver to regulate glucose output. Elucidation of this pathway may provide a novel target for the treatment of insulin resistant disorders such as diabetes mellitus.

\section{Methods}

Mice. Male mice were used in all experiments. The $I R^{\operatorname{lox} P / l o x P}$ and Foxol $1^{\operatorname{lox} P / \operatorname{lox} P}$

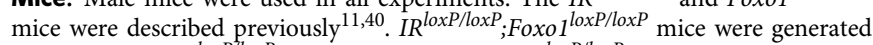
by crossing the $I R^{\text {loxPlloxP }}(\mathrm{C} 57 \mathrm{Bl} / 6 \mathrm{~J})$ with the Foxol $1^{\text {loxP }} /$ loxP $(\mathrm{FVB})$ mice. These mice were on a $129-\mathrm{C} 57 \mathrm{BL} / 6 \mathrm{~J}-\mathrm{FVB}$ mixed background. Mice were injected at 6- to 8 -weeks of age with $10^{11}$ genomic copies per mouse with adeno-associated-virus containing a liver-specific promoter, thyroxine-binding globulin (TBG) promoter driving either GFP or Cre recombinase to generate LWT, L-Insulin Receptor KO, or L-IRFoxolDKO ${ }^{8}$. The LWT group consisted of pooled GFP injected $I R^{\text {loxP } / l o x P}$ and $I R^{\text {loxP/loxP }}$;Foxo $1^{\text {loxP/loxP }}$ mice. GFP injected $I R^{\text {loxP/loxP }}$ and $I R^{\text {loxP/loxP }}$; Foxol ${ }^{\text {loxP }} /$ loxP mice did not significantly differ in any observed measurement therefore data is shown as the pooled averages from both genotypes. Experiments were performed 2-3 weeks after virus injection. To generate liver-specific congenital knockouts, $I R^{\operatorname{lox} P / l o x P}$ and $I R^{\text {loxP/loxP }} ;$ Foxo $1^{\text {loxP } / \text { loxP }}$ mice were crossed to 
an Alb-Afp-cre mouse ${ }^{41}$ (kind gift from Klaus Kaestner) and littermate floxed mice served as controls. For the fasting and refeeding experiments, mice were deprived of food for $16 \mathrm{~h}$ overnight, then killed (for the fasted groups) or refed with normal chow (Laboratory Rodent Diet 5,001) for an additional $4 \mathrm{~h}$ before killing (for the refed group). For insulin injection, 16-h fasted mice were i.p. injected with 3\% bovine serum albumin in saline or insulin at $2 \mathrm{mU} \mathrm{g}^{-1}$ of body weight $20 \mathrm{~min}$ before killing the mice and excising the liver for rapid freezing in liquid nitrogen. All mice experiments were reviewed and approved by the University of Pennsylvania Institutional Animal Care and Use Committee in accordance with the guidelines of the National Institutes of Health.

Liver protein extraction and western blotting. Livers were freeze clamped and stored at $-80^{\circ} \mathrm{C}$ until further processing. Protein lysates were extracted from frozen livers with a modified RIPA buffer $(150 \mathrm{mM} \mathrm{NaCl}, 50 \mathrm{mM}$ Tris, $\mathrm{pH} 7.6$, $1 \%$ Triton X-100, $0.5 \%$ sodium deoxycholate and $0.1 \%$ SDS, supplemented with protease (Roche) and phosphatase (Sigma) inhibitors. Cleared supernatants were extracted from cellular debris following 15 -min centrifugation at 17,000 $\mathrm{g}$. The following antibodies were used for immunoblotting at a dilution of 1:1,000: insulin receptor, IGF1-R, Foxo1, p-Ser 473 Akt, Akt, pPRAS 40, PRAS, p-S6 Ser 240 and Ser 244, S6 were from Cell Signaling Technology; antibody to Igfbp1 was purchased from Santa Cruz Biotechnology; antibodies to GFP and tubulin were from Sigma; antibody to Gck was provided by M.A. Magnuson (Vanderbilt University). Complete scans of key immunoblots are provided in Supplementary Fig. 1.

mRNA isolation and real-time PCR. Total RNA was isolated from frozen livers using the NucleoSpin RNA kit from Clontech. Complementary DNA was synthesized using Moloney murine leukaemia virus reverse transcriptase and the relative expression of the genes of interest was quantified by real-time PCR using the SYBR Green Dye-based assay. Primer sequences are provided in Supplementary Table 1 .

Primary hepatocytes isolation. Hepatocytes were isolated from random fed mice using a two-step collagenase/DNAse digestion protocol ${ }^{42}$. For glucose output studies, primary hepatocytes from indicated genotypes were isolated and plated in M199 media containing 10\% FBS, $100 \mathrm{nM}$ T3, $500 \mathrm{nM}$ dexamethasone and $1 \mathrm{nM}$ insulin. Following attachment, cells were changed to M199 media containing $100 \mathrm{nM}$ dexamethasone, and $1 \mathrm{nM}$ insulin were indicated and incubated overnight. Cells were washed switched to glucose output media (DMEM no glucose) and incubated with $10 \mathrm{mM}$ lactate $1 \mathrm{mM}$ pyruvate, $100 \mathrm{nM}$ dexamethasone and $100 \mathrm{nM}$ insulin and/or $100 \mathrm{uM} \mathrm{db}$-cAMP were indicated. Glucose production was determined by the measurement of glucose in the media over a $6 \mathrm{~h}$ time course using hexokinase glucose assay. Cells were lysed in modified RIPA buffer described above and subjected to western blot analysis or protein normalization for glucose output studies. Alternatively, cells were harvested for RNA isolation and subsequent gene expression analysis as described above.

Metabolic measurements. For glucose tolerance test, overnight fasted mice were given glucose at $2 \mathrm{~g} \mathrm{~kg}^{-1}$ body weight via intraperitoneal injection. Blood glucose concentrations in the mice were monitored by tail bleeding at $0,15,30,60$ and $120 \mathrm{~min}$ after glucose injection. Insulin concentrations were measured from plasma collected from the mice before and $15 \mathrm{~min}$ following glucose injection.

Hyperinsulinemic-euglycemic clamp. Euglycemic clamps were performed on unrestrained and awake 8-10-week-old mice ${ }^{6}$. Indwelling catheters were placed into mice 5-7 days prior to clamp experiments. Mice were fasted for $4 \mathrm{~h}$ and a priming $5 \mu \mathrm{Ci}$ [3-3H]glucose (Perkin Elmer HPLC purified) bolus followed by $0.05 \mu \mathrm{Ci} / \mathrm{min}$ infusion was initiated for $120 \mathrm{~min}$. Following the basal period, mice were given a bolus of $150 \mathrm{mU} \mathrm{kg}^{-1}$ insulin (or PBS) and a $0.1 \mu \mathrm{Ci} \mathrm{min}{ }^{-1}$ [3-3H]glucose infusion with insulin was initiated ( $6 \mathrm{~h}$ from start of fast). The rate of insulin infusion was $2.5 \mathrm{mU} \mathrm{kg}^{-1} \mathrm{~min}^{-1}$, and the glucose infusion rate was adjusted to keep euglycemic levels between 100 and $130 \mathrm{mg} \mathrm{dl}^{-1}$. PBS without glucose was infused in the control groups for gene expression analysis. Blood was taken by tail bleeding, and blood glucose levels were measured every $10 \mathrm{~min}$ during the clamp. Rd and HGP were calculated from the blood sampling every $10 \mathrm{~min}$ during the steady state portion of $(80-120 \mathrm{~min})$. At the end of the 2-h clamp, mice were killed by pentobarbital injection, and livers were quickly removed, freeze clamped in liquid nitrogen and stored at $-80^{\circ} \mathrm{C}$ for future uses.

Statistical analysis. All data are presented as mean \pm s.e.m. Statistical analysis was performed using one-way analysis of variance followed by Tukey post-test when more than two groups were compared, two-way analysis of variance followed by Bonferroni post-test when two conditions were involved and a Students's $t$ test with two-tailed analysis when only two groups of data were concerned. A $P<0.05$ was considered statistically significant.

\section{References}

1. Rizza, R. A. Pathogenesis of fasting and postprandial hyperglycemia in type 2 diabetes: implications for therapy. Diabetes 59, 2697-2707 (2010).

2. Saltiel, A. R. \& Kahn, C. R. Insulin signalling and the regulation of glucose and lipid metabolism. Nature 414, 799-806 (2001).

3. Lin, H. V. \& Accili, D. Hormonal regulation of hepatic glucose production in health and disease. Cell Metab. 14, 9-19 (2011).

4. Leavens, K. F. \& Birnbaum, M. J. Insulin signaling to hepatic lipid metabolism in health and disease. Crit. Rev. Biochem. Mol. Biol. 46, 200-215 (2011).

5. Menon, S. et al. Spatial control of the TSC complex integrates insulin and nutrient regulation of mTORC1 at the lysosome. Cell 156, 771-785 (2014).

6. Wan, M. et al. A noncanonical, GSK3-independent pathway controls postprandial hepatic glycogen deposition. Cell Metab. 18, 99-105 (2013).

7. Li, S., Brown, M. S. \& Goldstein, J. L. Bifurcation of insulin signaling pathway in rat liver: $\mathrm{mTORC1}$ required for stimulation of lipogenesis, but not inhibition of gluconeogenesis. Proc. Natl Acad. Sci. USA 107, 3441-3446 (2010).

8. Wan, M. et al. Postprandial hepatic lipid metabolism requires signaling through Akt2 independent of the transcription factors FoxA2, FoxO1, and SREBP1c. Cell Metab. 14, 516-527 (2011).

9. Nakae, J., Park, B. C. \& Accili, D. Insulin stimulates phosphorylation of the forkhead transcription factor FKHR on serine 253 through a Wortmanninsensitive pathway. J Biol Chem 274, 15982-15985 (1999).

10. Rena, G. et al. Two novel phosphorylation sites on FKHR that are critical for its nuclear exclusion. EMBO J. 21, 2263-2271 (2002).

11. Matsumoto, M., Pocai, A., Rossetti, L., Depinho, R. A. \& Accili, D. Impaired regulation of hepatic glucose production in mice lacking the forkhead transcription factor Foxol in liver. Cell Metab. 6, 208-216 (2007).

12. Dentin, R. et al. Insulin modulates gluconeogenesis by inhibition of the coactivator TORC2. Nature 449, 366-369 (2007).

13. Koo, S. H. et al. The CREB coactivator TORC2 is a key regulator of fasting glucose metabolism. Nature 437, 1109-1111 (2005).

14. Puigserver, P. et al. Insulin-regulated hepatic gluconeogenesis through FOXO1-PGC-1alpha interaction. Nature 423, 550-555 (2003).

15. Dong, X. C. et al. Inactivation of hepatic Foxol by insulin signaling is required for adaptive nutrient homeostasis and endocrine growth regulation. Cell Metab. 8, 65-76 (2008).

16. Samuel, V. T. et al. Targeting foxol in mice using antisense oligonucleotide improves hepatic and peripheral insulin action. Diabetes 55, 2042-2050 (2006).

17. Lu, M. et al. Insulin regulates liver metabolism in vivo in the absence of hepatic Akt and Foxol. Nat. Med. 18, 388-395 (2012).

18. Michael, M. D. et al. Loss of insulin signaling in hepatocytes leads to severe insulin resistance and progressive hepatic dysfunction. Mol. Cell 6, 87-97 (2000).

19. Cheng, Z. \& White, M. F. The AKTion in non-canonical insulin signaling. Nat. Med. 18, 351-353 (2012).

20. Haas, J. T. et al. Hepatic insulin signaling is required for obesity-dependent expression of SREBP-1c mRNA but not for feeding-dependent expression. Cell Metab. 15, 873-884 (2012).

21. Levine, R. \& Fritz, I. B. The relation of insulin to liver metabolism. Diabetes 5, 209-222 (1956).

22. Okamoto, H., Obici, S., Accili, D. \& Rossetti, L. Restoration of liver insulin signaling in Insr knockout mice fails to normalize hepatic insulin action. J. Clin. Invest. 115, 1314-1322 (2005).

23. Fisher, S. J. \& Kahn, C. R. Insulin signaling is required for insulin's direct and indirect action on hepatic glucose production. J. Clin. Invest. 111, 463-468 (2003).

24. LeRoith, D. \& Yakar, S. Mechanisms of disease: metabolic effects of growth hormone and insulin-like growth factor 1. Nat. Clin. Pract. Endocrinol. Metab. 3, 302-310 (2007).

25. Myers, Jr. M. G. \& Olson, D. P. Central nervous system control of metabolism. Nature 491, 357-363 (2012).

26. Gelling, R. W. et al. Insulin action in the brain contributes to glucose lowering during insulin treatment of diabetes. Cell Metab. 3, 67-73 (2006).

27. Schwartz, M. W. et al. Cooperation between brain and islet in glucose homeostasis and diabetes. Nature 503, 59-66 (2013).

28. Inoue, H. et al. Role of hepatic STAT3 in brain-insulin action on hepatic glucose production. Cell Metab. 3, 267-275 (2006).

29. Pocai, A., Obici, S., Schwartz, G. J. \& Rossetti, L. A brain-liver circuit regulates glucose homeostasis. Cell Metab. 1, 53-61 (2005).

30. Konner, A. C. et al. Insulin action in AgRP-expressing neurons is required for suppression of hepatic glucose production. Cell Metab. 5, 438-449 (2007)

31. Ramnanan, C. J. et al. Brain insulin action augments hepatic glycogen synthesis without suppressing glucose production or gluconeogenesis in dogs. J. Clin. Invest. 121, 3713-3723 (2011).

32. Ramnanan, C. J. et al. Interaction between the central and peripheral effects of insulin in controlling hepatic glucose metabolism in the conscious dog. Diabetes 62, 74-84 (2013). 
33. Lewis, G. F., Vranic, M., Harley, P. \& Giacca, A. Fatty acids mediate the acute extrahepatic effects of insulin on hepatic glucose production in humans. Diabetes 46, 1111-1119 (1997).

34. Rebrin, K., Steil, G. M., Mittelman, S. D. \& Bergman, R. N. Causal linkage between insulin suppression of lipolysis and suppression of liver glucose output in dogs. J. Clin. Invest. 98, 741-749 (1996).

35. Mittelman, S. D. \& Bergman, R. N. Inhibition of lipolysis causes suppression of endogenous glucose production independent of changes in insulin. Am. J. Physiol. Endocrinol. Metab. 279, E630-E637 (2000).

36. Kawamori, D. et al. Insulin signaling in alpha cells modulates glucagon secretion in vivo. Cell Metab. 9, 350-361 (2009).

37. Myers, S. R. et al. Effects of small changes in glucagon on glucose production during a euglycemic, hyperinsulinemic clamp. Metabolism 40, 66-71 (1991).

38. Yecies, J. L. et al. Akt stimulates hepatic SREBP1c and lipogenesis through parallel mTORC1-dependent and independent pathways. Cell Metab. 14, 21-32 (2011).

39. Zhou, Y. et al. Regulation of glucose homeostasis through a XBP-1-FoxO1 interaction. Nat. Med. 17, 356-365 (2011).

40. Bruning, J. C. et al. A muscle-specific insulin receptor knockout exhibits features of the metabolic syndrome of NIDDM without altering glucose tolerance. Mol. Cell 2, 559-569 (1998).

41. Parviz, F. et al. Hepatocyte nuclear factor 4alpha controls the development of a hepatic epithelium and liver morphogenesis. Nat. Genet. 34, 292-296 (2003).

42. Miller, R. A. et al. Adiponectin suppresses gluconeogenic gene expression in mouse hepatocytes independent of LKB1-AMPK signaling. J. Clin. Invest. 121, 2518-2528 (2011).

\section{Acknowledgements}

We thank D. Accili (Columbia University) for sharing the Foxol ${ }^{\text {loxP } / l o x P}$ mice and C. Ronald Kahn (Joslin) for sharing the $I R^{\operatorname{lox} P / l o x P}$ mice. This work was supported by the US National Institutes of Health grant R01 DK056886 (M.J.B.), NRSA individual postdoctoral fellowship F32 DK101175 (P.M.T.) and the Samuel Chiaffa Memorial Fund (P.M.T.)

\section{Author contributions}

P.M.T. conceived of the hypothesis, designed and performed the experiments and analysed the data. Q.C., B.R.M. provided technical assistance. M.J.B. conceived the hypothesis and directed the project. P.M.T. and M.J.B. prepared the manuscript.

\section{Additional information}

Supplementary Information accompanies this paper at http://www.nature.com/ naturecommunications

Competing financial interests: M.J.B is an employee of Pfizer, Inc. The remaining authors declare no competing financial interests.

Reprints and permission information is available online at http://npg.nature.com/ reprintsandpermissions/

How to cite this article: Titchenell, P. M. et al. Hepatic insulin signalling is dispensable for suppression of glucose output by insulin in vivo. Nat. Commun. 6:7078 doi: $10.1038 /$ ncomms 8078 (2015). 